\title{
A NEW APPROACH TO TARGETING VERIFICATIONS AT THE ROYAL BOTANIC GARDEN EDINBURGH
}

\author{
Rob Cubey \& Martin F. Gardner
}

\begin{abstract}
Verification is the process of identifying and accurately naming the plants in the Living Collections. The Royal Botanic Garden Edinburgh (RBGE) has had a well organised system for verifying plants in place for many years but, despite this, only $26 \%$ of the Living Collections has been verified. The process is slow and time consuming and is biased towards groups and geographical areas in which Garden staff have a research interest. In the last two years, however, a new, more targeted approach to verification, to run in tandem with the existing system, has been developed that is more timeefficient. With this approach herbarium material is collected for each accession and the whole group is verified in one intensive session. Trial runs have been conducted on Alnus and Acer to great effect and further tests are being conducted on Mahonia and Spiraea.
\end{abstract}

\section{INTRODUCTION}

The Living Collections at the Royal Botanic Garden Edinburgh (RBGE) could, by some, simply be regarded as the most public face of the Garden. However, the colourful displays that delight and amaze the general public are also fundamental to the Garden's core activities of research, education and conservation.

Most of the plants in the Collection have been painstakingly collected on carefully planned, overseas expeditions to remote and sometimes dangerous parts of the world, often at considerable expense. These plants have then been skillfully propagated and grown-on by specialist horticultural staff. Careful attention and considerable time has been devoted to recording their wild collection details as well as monitoring the treatments they have received while in the Collection. Yet, after all this effort and commitment, $74 \%$ of the Living Collections still await verification (verification is the process of ensuring that the name given to a plant is correct; the term identification is sometimes used in its place).

Like many other botanic gardens, RBGE is guilty of not giving a high enough priority to the verification of its Living Collections. One reason for this may be that the process of verifying the Collections is not seen as 'cutting-edge' science and as such does not easily fit into the project-based approach to research. Yet it is widely recognised that having an accurately identified reference collection of living plants

Robert Cubey is the Plant Records Officer at the Royal Botanic Garden Edinburgh.

Martin Gardner is Co-ordinator of the International Conifer Conservation Programme, based at the Royal Botanic Garden Edinburgh. 
provides an invaluable resource on which all other aspects of the Garden's work is dependent. While RBGE's level of verifying the Living Collections is perhaps higher than many other botanic gardens, nevertheless the fact remains that only $26 \%$ of the Living Collections at RBGE have been verified (10,759 of its 41,533 accessions). However, when consideration is given to the fact that accessions of known wild origin are prioritised, then this percentage rises slightly to $30 \%$ (6,436 of 22,400 accessions).

While around 850 accessions are verified each year, this hardly keeps pace with the number of new accessions coming into the Garden. Over a period of 24 years, new accessions have averaged 3,391 per year. Due to the frequently slow nature of plant growth there will always remain a percentage of the collection that cannot yet be verified, as accurate verification often requires flower and/or fruits.

The RBGE has recognised that these verification figures are low and has set ambitious targets to rectify the situation. This short paper describes one of the protocols that have been developed in an attempt to increase the percentage of verified accessions in the Living Collections.

\section{BACKGROUND TO VERIFICATION AT THE RBGE}

Probably the greatest level of activity concerning verification of the Collection coincided with the period in which RBGE was the secretariat for the European Garden Flora Project in the early 1980s. During this time material from the Living Collections that needed to be verified was collected each Wednesday by horticultural staff and taken to appropriate members of the scientific staff. Later in the day the resulting verifications were discussed in a group meeting of both scientific and horticultural members of staff. This ordered approach resulted in the identification of a wide range of plant material from throughout the Garden. The process, which was instigated by Dr James Cullen, then Deputy Regius Keeper of the Garden and Secretary of the European Garden Flora project, also helped to integrate staff from the Horticultural and Scientific Divisions and provided a strong element of training for all concerned.

The present-day routine is fairly similar but less formal. It involves accessions that are in need of verifying being selected by horticultural staff (sometimes in conjunction with a specific request by a member of the scientific staff) and taken to scientific staff according to their areas of geographic or taxonomic speciality.

In recent years the verification process has also been boosted by the publication of the RBGE Catalogue of Plants. This was particularly so with the 2001 publication (Govier et al, 2001), as the graph below shows.

Due to the amount of time that it can take to verify plants, there is a nominal limit of six accessions that can be sent in for verification from each of the main Departments of the Inverleith site; though this can be increased if prior warning is given. The problems associated with transporting fresh material from Edinburgh's Specialist Gardens (Benmore, Logan and Dawyck Botanic Gardens) leads to a more 


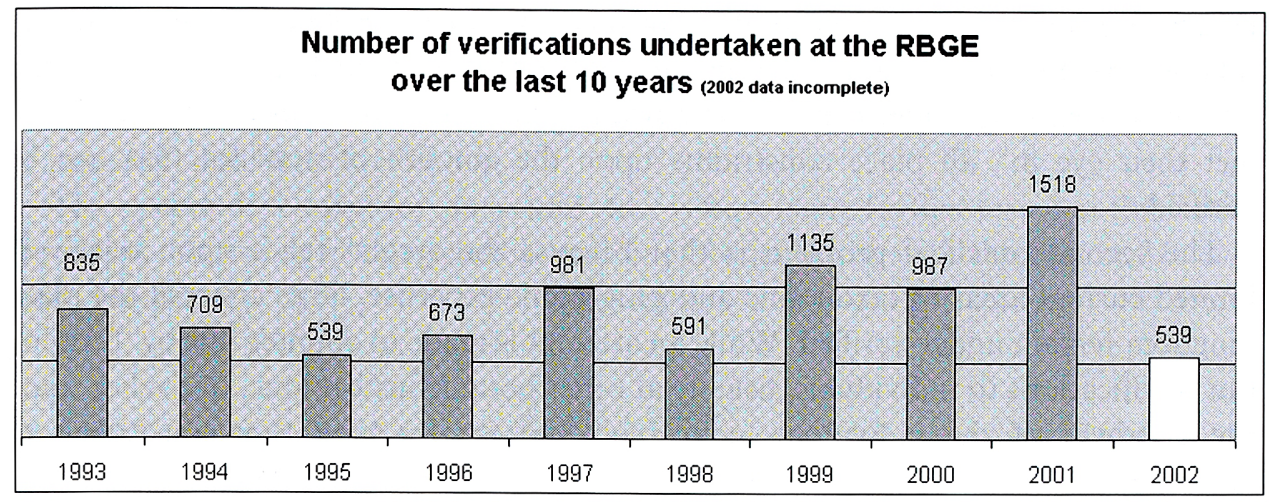

erratic schedule based more around when staff are able to travel to and from Edinburgh rather than on need. On average, however, over the past five years 24 accessions have been verified per week.

\section{THE PROCESS OF VERIFICATION}

The verification process of identifying and accurately naming plants is done in two steps. The first is to either confirm the existing name under which the plant originally came into the Garden or, if unknown, to determine the plant's identity. The second is to ensure that the name selected is valid and accepted by the wider botanic community.

However, with the backlog of unverified material in the Collection, RBGE needed to be able to prioritise material. After discussion the following unordered guidelines were issued giving guidance about the most worthwhile material to put forward for verification:

- Plants from accessions of known wild origin

- Plants from accessions that have not been identified to family

- Plants from accessions that have only been identified to generic level

- Plants from accessions that are of conservation importance including those listed by IUCN as being of conservation concern

- Groups in which RBGE has a particular research interest, e.g. Umbelliferae, Rhododendron, or temperate South American plants

- Plants from accessions suspected of being incorrectly named

- Plants that are used in publicity or for teaching material

- Plants that are recommended for de-accessioning

- Plants with a name change due to nomenclatural reasons

- Short lived, wild collected material

While this system rightly prioritises certain groups of plants for preferential treatment it can allow certain taxa to slip through the verification net because it contains two 
practical problems. The first is that it can take an unreasonably long time to undertake a 'one-off' verification. Time taken to locate the current taxonomic literature, access reference specimens in the herbarium and, importantly, for the scientist to 'get their eye in', all place constraints upon the amount of material that can be verified.

The second practical problem is that because the system relies upon relatively limited current areas of taxonomic and geographic expertise, large areas of the plant kingdom remain under-verified. While an attempt is made to counter this by 'farming out' verifications to individuals, usually at other botanic institutions, this relies upon the goodwill of the individuals concerned and is also very time-consuming to co-ordinate.

\section{THE GENUS-BASED APPROACH}

In an effort to counter these problems a new approach has been adopted by the Garden over the last two years to run alongside the existing system. Known informally as the genus-based approach to verification, it works by targeting a single genus that is in need of comprehensive verification. Over an allocated period of time (usually between one and three years) all accessions of the genus concerned are collected from the Living Collection and pressed. Then, at the end of the year the whole group can be verified at the same time.

This system has proved to be far more time-efficient than the ad hoc system. Since the genera have been agreed in advance, staff can ensure that all material collected contains the necessary diagnostic characteristics. It also runs over the length of the growing season so all growth forms including both flowering and fruiting characteristics can be collected if required for the verification process.

\section{TESTING THE APPROACH}

The protocols for the genus-based approach were first established by Simon Crutchley and Martin Gardner using the genus Alnus. During the summer of 2000 they verified 35 accessions of Alnus at the RBGE and, in the end, the actual verification process took only three to four hours. Amongst a lot of new information, it resulted in the discovery that four species were incorrectly named. Further refinement of the protocols were undertaken in 2001 when Richard Eden and Martin Gardner took on the much larger genus Acer. Two hundred and forty two accessions of Acer were verified and the exercise resulted in the confirmation that the vast majority (197) of the accessions were correctly named. However, it resulted in name changes for 35 accessions through identification or re-identification and a further ten accessions had their names changed due to synonymy. Two accessions were found to be dead and four accessions could not be verified due to a lack of diagnostic characters.

The collection and drying of the 302 specimens that were gathered took around 
ten days in total. The review of the taxonomic literature took another day, and the curation of the existing reference material in the herbarium took a further ten days. The actual verification of the 302 specimens once the background work had been completed took three weeks.

The system developed for Alnus and Acer is now being further tested at the RBGE by teams consisting of one scientist and one horticulturist with the genera Mahonia and Spiraea.

\section{CONClusions}

With the genus-based approach verifications are undertaken en-masse. The specimens (reference material) from the herbarium can be laid out for easy comparison, all necessary taxonomic literature can be assembled and the researchers can become familiar with the genus while undertaking the verifications - all factors that greatly speed up the process. The approach also provides the opportunity for a short, intensive period for staff training (one or two days) with extremely productive outputs.

Obviously this approach does entail a relatively large amount of effort being invested in a short period of time, not only to identify the material but also in the process of collecting and drying the plants (in the case of RBGE this can include collections being made from the four widely geographically distributed Specialist Gardens). There are also other important processes that form part of the approach. One of these is the opportunity to curate the herbarium specimens for the genus under investigation so that the identifications can take place. Another positive outcome is that, once verified, the material can be mounted and be added to the cultivated herbarium.

Over the last ten years 77 staff, including specialists from other institutions, have been involved in verifying the Living Collections at RBGE. In so doing they have contributed greatly to the verification process and helped to influence the new protocols which are being adopted today. This in turn will help the Garden achieve its new ambitious verification level of a $2 \%$ increase per year

\section{REFERENCES}

Govier et al (2001). Catalogue of Plants 2001. Royal Botanic Garden Edinburgh. Thomas \& Watson. (2000). Royal Botanic Garden Data Management for Plant Collections - A Handbook of Best Practice. Royal Botanic Garden Edinburgh. 
